\title{
Pulse Arrival Time Accurately Detects Pacing-Induced Mechanical Alternans
}

\author{
Stefan van Duijvenboden ${ }^{1}$, Nick Child ${ }^{2}$, Jaswinder S. Gill ${ }^{2}$, Ben Hanson ${ }^{3}$, Peter Taggart ${ }^{1,2}$, Michele \\ Orini $^{1,3}$ \\ ${ }^{1}$ Institute of Cardiovascular Science, UCL, London, UK \\ ${ }^{2}$ Department of Cardiology, Guy's \& St Thomas' Hospitals NHS Foundation Trust, London, UK \\ ${ }^{3}$ Department of Mechanical Engineering, UCL, London, UK
}

\begin{abstract}
Accurate cardiac risk stratification is crucial for preventing cardiac death, but still remains an unmet need. Mechanical alternans (MA), an oscillation of blood pressure that occurs on a beat-to-beat basis, has been recognized as a marker of cardiac instability and is associated with an increased risk of cardiac death. However, the use of MA for risk stratification is currently limited by the invasiveness and costs of continuous blood pressure monitoring. A non-invasive, cuffless and affordable method to detect MA is therefore highly desirable. The pulse transit time (PTT) and the pulse arrival time (PAT) are promising techniques for continuous blood pressure monitoring, particularly for detecting short-term blood pressure changes. In this work, we hypothesized that PAT, measured as the interval between the R-wave in the ECG and a distal arterial pulse, can be used to accurately track fast beat-to-beat blood pressure dynamics and detect MA.

A total of 42 ECG and femoral arterial pressure recordings from 12 patients with normal ventricles were analyzed. Patients were instructed to breath at a fixed respiratory rate and MA was induced by ventricular pacing. Both MA and PAT alternans were detected using spectral analysis. MA was present in $69 \%$ of blood pressure recordings $(n=29)$. ROC analysis showed that PAT accurately detected MA, with an area under the curve was equal to $\mathrm{AUC}=0.94$. The optimal threshold for detecting MA by using PAT provided $90 \%$ sensitivity and $85 \%$ specificity. In conclusion, this study demonstrates that PAT can be used to accurately detect pacing-induced MA and may represent a first step toward non-invasive, cuff-less and affordable MA screening for cardiac risk assessment.
\end{abstract}

\section{Introduction}

Cardiac alternans refers to either mechanical or electrical oscillations that occur on a beat-to-beat basis [1]. Electrical alternans generates at the cardiac myocyte level where it manifests as an oscillation of the action potential duration [2] and can be detected on the surface ECG as a beat-to-beat change in the T-wave morphology [3]. T-wave alternans is a well-established non-invasive marker of cardiac instability and a predictor of sudden cardiac death $[3,4]$. Mechanical alternans (MA), first observed in the XIX century [1], usually refers to a repetitive sequence of strong and weak pulses and has been recognized as a marker of cardiac instability. Recent studies have shown that MA is associated with cardiac death in heart failure [5] and dilated cardiomyopathy [6] patients.

Accurate risk stratification in cardiac disease remains an unmet need, with both serious social and economic implications. At present, despite encouraging results $[5,6]$, the assessment and utilization of MA as a marker for cardiac risk stratification is limited by the invasiveness of continuous blood pressure monitoring and by the cost associated with invasive recordings. Therefore, a non-invasive and affordable method for detecting MA is needed to conduct studies to assess the prediction values of MA at a population level, with possibly great potential in terms of cardiac death prevention.

The Pulse transit time (PTT) and pulse arrival time (PAT) are promising non-invasive techniques for continuous blood pressure monitoring, particularly for detecting short-term perssure changes $[7,8]$. The PTT, defined as the the time delay for the pressure wave to travel between two arterial sites, and the PAT, defined as the interval between the R-wave in the ECG and a distal arterial pulse, are both negatively associated with blood pressure [9]. In recent years, photoplethysmography has provided the opportunity of measuring PAT from the body surface, therefore offering the possibility of non-invasive, cuff-less and affordable blood pressure monitoring. Although the accuracy of (absolute) blood pressure estimates based on PTT and PAT is controversial [10], we hypothesize that both methods can be used to track fast beat-to-beat blood pressure dynamics and detect MA. To the best of our knowledge the association between PAT and MA has never been studied. Therefore, this work aims to assess a novel and simple 
methodology to detect MA based on PAT in a database of patients with normal ventricles undergoing electrophysiological studies including surface ECG and invasive blood pressure monitoring. We consider this study a first step toward non-invasive, cuff-less and affordable MA screening.

\section{Methods}

\section{Data collection}

Studies were performed in 12 patients (10 males, 2 females, aged 4868, median 54) during in the unsedated state before the routine clinical procedure for radiofrequency ablation for atrial fibrillation at St Thomas' Hospital, London, as described previously $[11,12]$. None of the subjects was known to have ventricular scar or disordered conduction due to bundle branch abnormality. Cardioactive medications were discontinued for 5 days before the study. Invasive arterial blood pressure was measured using a continuous-flush pressure transducer system (Tru-Wave PX600F; Ed- wards Lifesciences, Irvine, CA). Both limb leads ECGs and arterial blood pressure were recorded at a sampling frequency of $1200 \mathrm{~Hz}$. During the study, heart rate was clamped by ventricular pacing at a fixed cycle length (median, $500 \mathrm{~ms}$ ) from the right ventricular apex using a Biotronik (Berlin, Germany) stimulator (model UHS 3000). Patients were breathing at four fixed rates (6, 9,15 , and 30 breaths/min) for $90 \mathrm{~s}$ each, in random order.

\section{Parameter extraction}

ECG and blood pressure signals were band-pass filtered between 0.5 and $35 \mathrm{~Hz}$, and ECG R-waves were identified and systolic blood pressure, $\operatorname{SBP}(n)$, measured for each heartbeat $n$. The pulse arrival time, $P A T(n)$, was measured as the time between the R-wave peak and the maximum first derivative of the upstroke in the pulse of the same heartbeat (see Fig. 1).

\section{Detection of mechanical and PAT alternans}

The presence of alternans was investigated using spectral analysis, as described previously $[13,14]$. The first step of this method is to apply a detrending filter to enhance changes in the SBP and PAT signal on a beat-to-beat basis:

$$
y(n)=S B P(n+1)-S B P(n)
$$

Next, spectral analysis was applied to quantify the magnitude of the oscillation that appears every other beat, i.e. at a frequency equal to 0.5 cycles per beat [13]. The spectrum
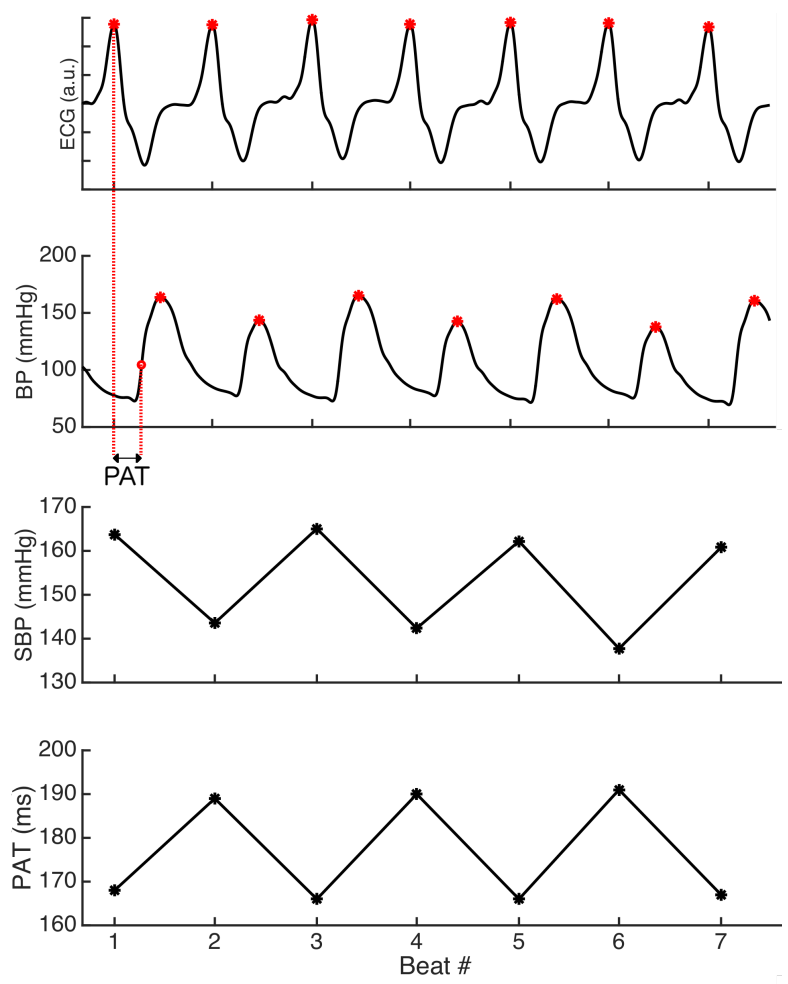

Figure 1. Representative example of pulse arrival time (PAT) alternans occurring as the same time as mechanical alternans. PAT was measured from the R-wave to the maximum upstroke of the corresponding pulse.

was estimated in a moving window of length $L=64$ beats:

$$
P_{\mathrm{SBP}}(n, f)=\left|\sum_{l=-L / 2+1}^{l / 2} y(n+l) e^{-2 \pi i l}\right|^{2}
$$

A SBP recording was classified as exhibiting MA if $P_{\mathrm{SBP}}(n, f=0.5)>4 \mathrm{mmHg}^{2}$, i.e. if the magnitude of MA was higher than $2 \mathrm{mmHg}$ in at least one sliding window.

PAT alternans was computed as in (1)-(2), but using $P A T(n)$ time series instead of $S B P(n)$. The optimum threshold to detect MA based on PAT alternans was established by ROC analysis, scanning PAT alternans magnitude from 1 to $30 \mathrm{~ms}$.

\section{Statistical analysis}

True positvie and false positive detections were defined as when PAT alternans was or was not detected in recordings exhibiting significant MA, respectively. False positive and negative detections were defined as when PAT alternans was or was not detected in recordings not exhibiting significant MA. The confusion matrix was constructed and 


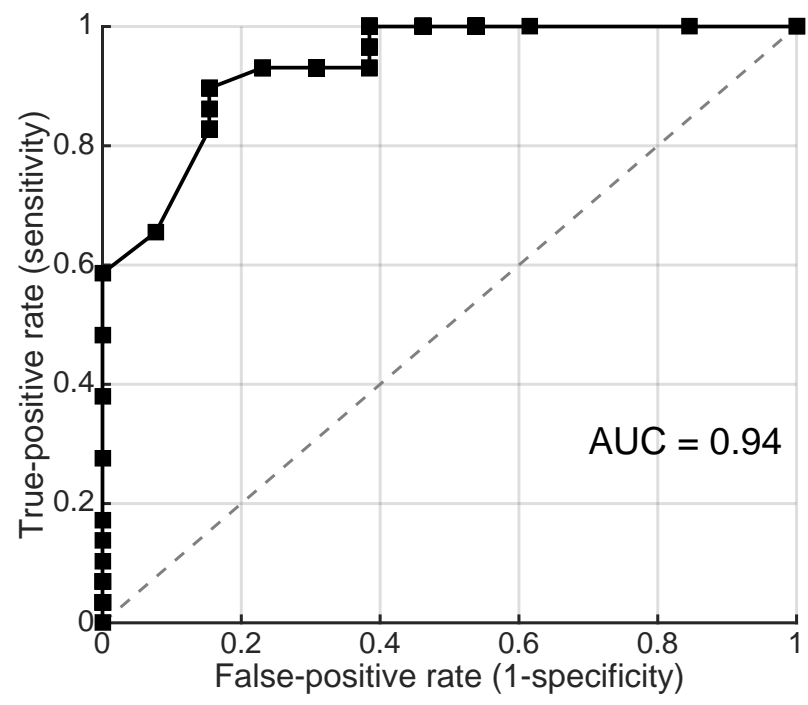

Figure 2. ROC curve showing the accuracy of PAT to predict mechanical alternans.

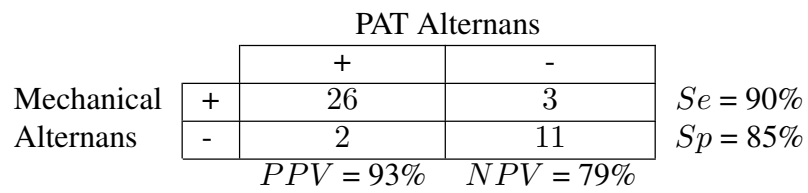

Table 1. Confusion matrix for MA detection using PAT for the optimal detection threshold. PPV: Positive Predictive Value, NPV: Negative Predictive Value, Se: Sensitivity, Sp: Specificity.

sensitivity, specificity, positive and negative predictive values as well as total accuracy were estimated.

\section{Results}

A total of 42 ECGs and blood pressure recordings from 12 patients were analyzed. Each recording was $90 \mathrm{~s}$ long and the respiratory frequency was fixed. The mean SBP was $137 \pm 19 \mathrm{mmHg}$. MA was observed in 29 recordings (69\%). The mean MA magnitude was $4.1 \mathrm{mmHg}$ (range: $2.1-12.8 \mathrm{mmHg}$ ). Figure 2 shows the ROC curve for MA detection using PAT. The area under the curve was AUC $=0.94$ and the optimal threshold (PAT alternans magnitude) for detecting MA was equal to $3.1 \mathrm{~ms}$, which provided a $90 \%$ sensitivity, $85 \%$ specificity and an accuracy of $88 \%$. The positive and negative predictive values (PPV \& NPV) were 93\% and 79\%, respectively. For this threshold, the mean PAT alternans magnitude was $5.3 \mathrm{~ms}$ (range: 3.1 - $13.6 \mathrm{~ms}$ ). The corresponding confusion matrix for this threshold is shown in Table 1.

\section{Discussion}

To the extent of our knowledge, this study is the first to demonstrate that the PAT can accurately detect pacing induced MA. This is an important finding since MA has been linked with poor prognosis, ischemia, disordered left ventricular function and cardiac arrhythmias [15]. In a prospective study conducted by Hirashiki et al, pacing induced MA was identified as an important prognostic factor for poor outcome in patients with idiopathic dilated cardiomyopathy [6]. PAT and PTT have already shown to conceivably permit continuous, noninvasive, and cuffless $\mathrm{BP}$ monitoring in the acute setting. The results of this study strongly suggests that non-invasive measurements based on PAT and potentially PTT may be utilised to improve cardiac risk stratification by enabling non-invasive and cuff-less monitoring of MA.

Although the exact mechanism underlying MA is not fully understood, there is general consensus that MA is associated with beat-to-beat variation of the left ventricular stroke volume, either caused by changes in myocardial contraction or left end-diastolic volume $[16,17]$ probably due to calcium handling imbalance [1]. In this study, MA was detected by measuring the systolic arterial pressure for each beat. To investigate whether estimation of MA could be improved, we extended our analysis by extracting multiple data points from the arterial waveform instead of only a single systolic value. This provided similar results. The presented work is based on invasive arterial blood pressure measurements. Further work is needed to determine whether PAT measured through photoplethysmography can be used to accurately detect MA and whether the methodology proposed in this paper can be also utilised to detect MA in sinus rhythm.

\section{Conclusion}

This work demonstrates that simple spectral analysis of the PAT can provide accurate detection of pacing-induced MA. This findings has implications for screening of MA for cardiac risk assessment.

\section{References}

[1] Laurita KR, Rosenbaum DS. Cellular mechanisms of arrhythmogenic cardiac alternans. Prog Biophys Mol Biol 2008;97(2-3):332-347.

[2] Zhou X, Bueno-Orovio A, Orini M, Hanson B, Hayward M, Taggart P, Lambiase PD, Burrage K, Rodriguez B. In Vivo and in Silico Investigation into Mechanisms of Frequency Dependence of Repolarization Alternans in Human Ventricular Cardiomyocytes. Circulation Research 2016; 118(2):266-278. ISSN 15244571.

[3] Verrier RL, Klingenheben T, Malik M, El-Sherif N, Exner DV, Hohnloser SH, Ikeda T, Martínez JP, Narayan SM, 
Nieminen T, Rosenbaum DS. Microvolt T-wave alternans: Physiological basis, methods of measurement, and clinical utilityconsensus guideline by international society for Holter and noninvasive Electrocardiology. Journal of the American College of Cardiology ;(13):1309-1324. ISSN 07351097.

[4] Bloomfield DM, Steinman RC, Namerow PB, Parides M, Davidenko J, Kaufman ES, Shinn T, Curtis A, Fontaine J, Holmes D, Russo A, Tang C, Bigger JT. Microvolt T-Wave Alternans Distinguishes Between Patients Likely and Patients Not Likely to Benefit From Implanted Cardiac Defibrillator Therapy. Circulation 2004;110(14).

[5] Kim R, Cingolani O, Wittstein I, McLean R, Han L, Cheng K, Robinson E, Brinker J, Schulman SS, Berger RD, Henrikson CA, Tereshchenko LG ;.

[6] Hirashiki A, Izawa H, Somura F, Obata K, Kato T, Nishizawa T, Yamada A, Asano H, Ohshima S, Noda A, Iino S, Nagata K, Okumura K, Murohara T, Yokota M ;.

[7] Gribbin B, Steptoe A, Sleight P. Pulse wave velocity as a measure of blood pressure change. Psychophysiology Jan 1976;13(1):86-90.

[8] Ma HT, Zhang YT. Spectral analysis of pulse transit time variability and its coherence with other cardiovascular variabilities. Conf Proc IEEE Eng Med Biol Soc 2006;1:64426445.

[9] Mukkamala R, Hahn JO, Inan OT, Mestha LK, Kim CS, Toreyin H, Kyal S. Toward Ubiquitous Blood Pressure Monitoring via Pulse Transit Time: Theory and Practice. IEEE Transactions on Biomedical Engineering aug; (8):1879-1901. ISSN 15582531.

[10] Zhang G, Gao M, Xu D, Olivier NB, Mukkamala R. Pulse arrival time is not an adequate surrogate for pulse transit time as a marker of blood pressure. Journal of Applied Physiology dec;(6):1681-1686. ISSN 8750-7587.

[11] Hanson B, Gill J, Western D, Gilbey MP, Bostock J, Boyett MR, Zhang H, Coronel R, Taggart P. Cyclical modulation of human ventricular repolarization by respiration. Frontiers in Physiology ;379. ISSN 1664042X.

[12] van Duijvenboden S, Hanson B, Child N, Orini M, Rinaldi CA, Gill J, Taggart P. Effect of autonomic blocking agents on the respiratory related oscillations of ventricular action potential duration in humans. American journal of physiology Heart and circulatory physiology 2015;309(12):ajpheart.00560.2015. ISSN 1522-1539 (Electronic).

[13] Martínez JP, Olmos S, Martinez JP, Olmos S. Methodological principles of $\mathrm{T}$ wave alternans analysis: A unified framework. IEEE Transactions on Biomedical Engineering 2005;52(4):599-613. ISSN 00189294.

[14] Orini M, Hanson B, Monasterio V, Martínez JP, Hayward M, Taggart P, Lambiase P. Comparative evaluation of methodologies for T-wave alternans mapping in electrograms. IEEE Transactions on Biomedical Engineering 2014;61(2):308-316. ISSN 00189294.

[15] Schaefer S, Malloy CR, Schmitz JM, Dehmer GJ. Clinical and hemodynamic characteristics of patients with inducible pulsus alternans. Am Heart J Jun 1988;115(6):1251-1257.

[16] Miller WP, Liedtke AJ, Nellis SH. End-systolic pressure- diameter relationships during pulsus alternans in intact pig hearts. Am J Physiol Apr 1986;250(4 Pt 2):H606-611.

[17] Gleason WL, Braunwald E. Studies on Starling's law of the heart. VI. Relationships between left ventricular enddiatolic volume and stroke volume in man with observations on the mechanism of pulsus alternans. Circulation May 1962; 25:841-848.

Address for correspondence:

Stefan van Duijvenboden, Institute of Cardiovascular Science, UCL, London WC1E 6BT, UK (e-mail: ucemvan@ucl.ac.uk) 